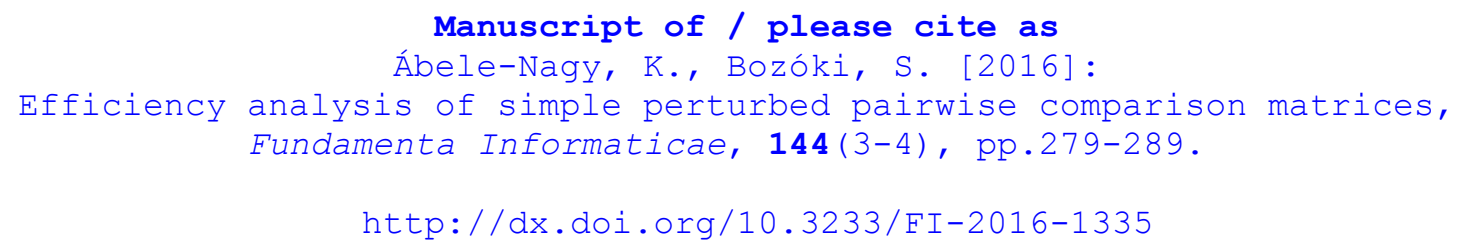

\title{
Efficiency analysis of simple perturbed pairwise comparison matrices
}

\author{
Kristóf Ábele-Nagy \\ Laboratory on Engineering and Management Intelligence, \\ Research Group of Operations Research and Decision Systems, \\ Institute for Computer Science and Control, \\ Hungarian Academy of Sciences (MTA SZTAKI); \\ Department of Operations Research and Actuarial Sciences, \\ Corvinus University of Budapest, Hungary \\ abele-nagy.kristof@sztaki.mta.hu \\ Sándor Bozóki ${ }^{1}$ \\ Laboratory on Engineering and Management Intelligence, \\ Research Group of Operations Research and Decision Systems, \\ Institute for Computer Science and Control, \\ Hungarian Academy of Sciences (MTA SZTAKI); \\ Department of Operations Research and Actuarial Sciences, \\ Corvinus University of Budapest, Hungary \\ bozoki.sandor@sztaki.mta.hu \\ http://www.sztaki.mta.hu/\%7Ebozoki
}

\begin{abstract}
Efficiency, the basic concept of multi-objective optimization is investigated for the class of pairwise comparison matrices. A weight vector is called efficient if no alternative weight vector exists such that every pairwise ratio of the latter's components is at least as close to the corresponding element of the pairwise comparison matrix as the one of the former's components is, and the latter's approximation is strictly better in at least one position. A pairwise comparison matrix is called simple perturbed if it differs from a consistent pairwise comparison matrix in one element and its reciprocal. One of the classical weighting methods, the eigenvector method is analyzed. It is shown in the paper that the principal right eigenvector of a simple perturbed pairwise comparison matrix is efficient. An open problem is exposed: the search for a necessary and sufficient condition of that the principal right eigenvector is efficient.
\end{abstract}

Keywords: pairwise comparison matrix, efficiency, Pareto optimality, eigenvector

\footnotetext{
${ }^{1}$ corresponding author
} 


\section{Introduction}

To determine the importance and/or the weights of criteria as well as comparing the alternatives in multi-attribute decision making problems are of crucial importance.

A ratio scale matrix $\mathbf{A}=\left[a_{i j}\right]$ is a positive square matrix with the reciprocal property $a_{i j}=1 / a_{j i}$, where $i, j=1,2, \ldots, n$, and $a_{i i}=1, i=1,2, \ldots, n$. An $a_{i j}$ entry from $\mathbb{R}$ in $\mathbf{A}$ represents the strength or the relative importance ratio of decision alternative $i$ over alternative $j$ with respect to a common criterion. The relative importance ratios are usually elicited from people who produce $n(n-1) / 2$ subjective judgments on each possible pair of the alternatives. Once such a matrix called pairwise comparison matrix (PCM) has been constructed ( $n \geq 3$ ), the objective is to extract the implicit (positive) weights of the various alternatives.

It is often easier to make these comparisons in pairs, by answering the questions

- How many times is a criterion more important than another criterion?

- With respect to a given criterion, how many times is an alternative better than another alternative?

- How many times is a voting power of a decision maker is greater than that of another decision maker?

- How many times is a scenario more probable than another one?

Numerical answers can be arranged in a matrix. Pairwise comparison matrix $\mathbf{A}=\left[a_{i j}\right]_{i, j=1, \ldots, n}$ thus has the following properties: $a_{i j}>0, a_{j i}=1 / a_{i j}$, $i, j=1,2, \ldots, n$. Let $\mathcal{P C} \mathcal{M}_{n}$ denote the class of pairwise comparison matrices of size $n \times n$, where $n \geq 3$. $\mathbf{A}$ is called consistent, if $a_{i k} a_{k j}=a_{i j}$ holds for all $i, j, k=1,2, \ldots, n$, otherwise it is called inconsistent.

Once the decision makers have provided their assessments on the pairwise ratios, the objective is to find a weight vector $\mathbf{w}=\left(w_{1}, w_{2}, \ldots, w_{n}\right)^{\top}$ such that these ratios, $\frac{w_{i}}{w_{j}}$ be found that are as close as possible to $a_{i j}$ s for all $i, j=$ $1,2, \ldots, n$. The column vector $\mathbf{w}$ is usually normalized so that $\sum_{i} w_{i}=1$. A weight vector can be extracted from a PCM in several ways [1, 6, 9, 13], however, the paper investigates the eigenvector method [14] only.

If $\mathbf{A}$ is consistent, then the eigenvector equation $\mathbf{A} \mathbf{w}=n \mathbf{w}$ holds, and $w_{i} / w_{j}$ $=$ $i, j=1,2, \ldots, n, w_{i}>0, \sum_{i} w_{i}=1$ [14]. This idea is extended to the general case as follows. The eigenvector method, proposed by Saaty [14], obtains the 
weight vector from the eigenvector equation $\mathbf{A} \mathbf{w}^{E M}=\lambda_{\max } \mathbf{w}^{E M}$, where $\lambda_{\max }$ denotes the Perron-eigenvalue (also known as maximal or principal eigenvalue) of $\mathbf{A}$, and $\mathbf{w}^{E M}$ is the corresponding principal right eigenvector.

$$
\lambda_{\max } \geq n
$$

and equality holds if and only if $\mathbf{A}$ is consistent. $\mathbf{w}^{E M}$ is positive and unique up to a scalar multiplication. Note that $\lambda_{\max }$ shall also be denoted by $\lambda_{\delta}$ in the beginning of Section 2 in order to emphasize its dependence on parameter $\delta$. Later on $\lambda_{\delta}$ shall be shortened by $\lambda$. Once $\mathbf{w}^{E M}$ is computed, the ratio $\frac{w_{i}^{E M}}{w_{j}^{E M}}$ can be compared to $a_{i j}$. In the consistent case, as mentioned before, $\frac{w_{i}^{E M}}{w_{j}^{E M}}=a_{i j}$ for all $i, j=1,2, \ldots, n$.

We now introduce the term efficiency also known as Pareto optimality, or nondominatedness [15, Chapter 2.4] which is considered to be a basic concept of multi-objective optimization and referring to the weight vectors derived from PCMs. Let $\mathbf{A}=\left[a_{i j}\right]_{i, j=1, \ldots, n} \in \mathcal{P C} \mathcal{M}_{n}$ and $\mathbf{w}=\left(w_{1}, w_{2}, \ldots, w_{n}\right)^{\top}$ be a positive weight vector.

Definition 1. A positive weight vector $\mathbf{w}$ is called efficient if no other positive weight vector $\mathbf{w}^{\prime}=\left(w_{1}^{\prime}, w_{2}^{\prime}, \ldots, w_{n}^{\prime}\right)^{\top}$ exists such that

$$
\begin{array}{ll}
\left|a_{i j}-\frac{w_{i}^{\prime}}{w_{j}^{\prime}}\right| \leq\left|a_{i j}-\frac{w_{i}}{w_{j}}\right| \quad \text { for all } 1 \leq i, j \leq n, \\
\left|a_{k \ell}-\frac{w_{k}^{\prime}}{w_{\ell}^{\prime}}\right|<\left|a_{k \ell}-\frac{w_{k}}{w_{\ell}}\right| \quad \text { for some } 1 \leq k, \ell \leq n .
\end{array}
$$

A weight vector $\mathbf{w}$ is called inefficient if it is not efficient.

It follows from the definition, that $\mathbf{w}^{E M}$ is efficient for every consistent PCM as $a_{i j}=\frac{w_{i}^{E M}}{w_{j}^{E M}}$ for all $i, j=1, \ldots, n$.

Blanquero et al. [2] investigated several necessary and sufficient conditions on efficiency. One of these efficiency conditions is utilized in our paper, which applies a directed graph representation.

Definition 2. Let $\mathbf{A}=\left[a_{i j}\right]_{i, j=1, \ldots, n} \in \mathcal{P C} \mathcal{M}_{n}$ and $\mathbf{w}=\left(w_{1}, w_{2}, \ldots, w_{n}\right)^{\top}$ be a positive weight vector. A directed graph $G:=(V, \vec{E})_{\mathbf{A}, \mathbf{w}}$ is defined as follows: $V=\{1,2, \ldots, n\}$ and

$$
\vec{E}=\left\{\operatorname{arc}(i \rightarrow j) \mid \frac{w_{i}}{w_{j}} \geq a_{i j}, i \neq j\right\} .
$$


Theorem 1. [2, Corollary 10] Let $\mathbf{A} \in \mathcal{P C M}_{n}$. A weight vector $\mathbf{w}$ is efficient if and only if $G=(V, \vec{E})_{\mathbf{A}, \mathbf{w}}$ is a strongly connected digraph, that $i s$, there exist directed paths from $i$ to $j$ and from $j$ to $i$ for all pairs of nodes $i, j$.

Blanquero et al. [2, Section 3] also showed that the principal right eigenvector can be inefficient. This remarkable result was recalled by Bajwa, Choo and Wedley [1] and by Conde and Pérez [7] and also by Fedrizzi [12].

Another numerical example is provided here to illustrate (in)efficiency and its digraph representation.

Example 1. Let $\mathbf{A} \in \mathcal{P C} \mathcal{M}_{4}$ as follows:

$$
\mathbf{A}=\left(\begin{array}{cccc}
1 & 1 & 1 / 5 & 1 / 5 \\
1 & 1 & 1 / 3 & 1 / 7 \\
5 & 3 & 1 & 1 / 4 \\
5 & 7 & 4 & 1
\end{array}\right)
$$

The principal right eigenvector of $\mathbf{A}$ and the consistent approximation of A, generated by $\mathbf{w}^{E M}$, are displayed, truncated at 8 and 4 correct digits, respectively:

$$
\mathbf{w}^{E M}=\left(\begin{array}{c}
0.07777933 \\
0.07732534 \\
0.24353753 \\
0.60135778
\end{array}\right), \quad\left[\frac{w_{i}^{E M}}{w_{j}^{E M}}\right]=\left(\begin{array}{cccc}
1 & 1.0058 & 0.3193 & 0.1293 \\
0.9941 & 1 & 0.3175 & 0.1285 \\
3.1311 & 3.1495 & 1 & 0.4049 \\
7.7315 & 7.7769 & 2.4692 & 1
\end{array}\right) .
$$

Let us apply Definition 2 in order to draw the digraph associated to matrix $\mathbf{A}$ and its principal right eigenvector $\mathbf{w}^{E M}$. The digraph in Figure 1 cannot be strongly connected because no arc leaves node 2.

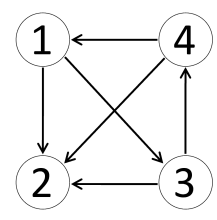

Figure 1. The principal right eigenvector in Example 1.1 is inefficient, because its associated digraph is not strongly connected

It might be instructive to see another, direct evidence why $\mathbf{w}^{E M}$ is inefficient. Let us increase the second coordinate of $\mathbf{w}^{E M}$ until it reaches $w_{1}^{E M}$, i.e., define 
$\mathbf{w}^{\prime}:=\left(w_{1}^{E M}, w_{1}^{E M}, w_{3}^{E M}, w_{4}^{E M}\right)^{\top}$. Then

$\mathbf{w}^{\prime}:=\left(\begin{array}{c}0.07777933 \\ 0.07777933 \\ 0.24353753 \\ 0.60135778\end{array}\right), \quad\left[\frac{w_{i}^{\prime}}{w_{j}^{\prime}}\right]=\left(\begin{array}{cccc}1 & \mathbf{1} & 0.3193 & 0.1293 \\ \mathbf{1} & 1 & \mathbf{0 . 3 1 9 3} & \mathbf{0 . 1 2 9 3} \\ 3.1311 & \mathbf{3 . 1 3 1 1} & 1 & 0.4049 \\ 7.7315 & \mathbf{7 . 7 3 1 5} & 2.4692 & 1\end{array}\right)$.

It can be seen that (with $\mathbf{w}=\mathbf{w}^{E M}$ ) the strict inequality (3) in Definition 1 holds exactly for the non-diagonal elements of the second row/column, marked by bold. For all other entries inequality (2) holds with equality.

Example 1.1 above illustrates that Theorem 1 is powerful and easy to apply.

Bozóki [3] showed that the principal right eigenvector of the parametric pairwise comparison matrix

$$
\mathbf{A}(p, q)=\left(\begin{array}{ccccccc}
1 & p & p & p & \ldots & p & p \\
1 / p & 1 & q & 1 & \ldots & 1 & 1 / q \\
1 / p & 1 / q & 1 & q & \ldots & 1 & 1 \\
\vdots & \vdots & \vdots & \ddots & & \vdots & \vdots \\
\vdots & \vdots & \vdots & & \ddots & \vdots & \vdots \\
1 / p & 1 & 1 & 1 & \ldots & 1 & q \\
1 / p & q & 1 & 1 & \ldots & 1 / q & 1
\end{array}\right) \in \mathcal{P C M}_{n}
$$

where $n \geq 4, p>0$ and $1 \neq q>0$, is inefficient.

However, the general problem is still open:

Question 1.1. What is the necessary and sufficient condition of that the principal right eigenvector is efficient?

Our significantly more moderate objective is to provide a new sufficient condition. The main contribution of the paper is the efficiency analysis of another special class of PCMs. Departing from a consistent PCM, let us modify a single element and its reciprocal, which results in a simple perturbed PCM. It will be shown in this paper that the principal right eigenvector of a simple perturbed pairwise comparison matrix is efficient. 


\section{Simple perturbed pairwise comparison ma- trix}

Let $x_{1}, x_{2}, \ldots, x_{n-1}$ be arbitrary positive numbers. Let $\delta$ represent a multiplicative perturbation factor with an arbitrary positive number, where $\delta \neq 1$. A simple perturbed PCM is defined as follows:

$$
\mathbf{A}_{\delta}=\left(\begin{array}{ccccc}
1 & x_{1} \delta & x_{2} & \ldots & x_{n-1} \\
\frac{1}{x_{1} \delta} & 1 & \frac{x_{2}}{x_{1}} & \ldots & \frac{x_{n-1}}{x_{1}} \\
\frac{1}{x_{2}} & \frac{x_{1}}{x_{2}} & 1 & \ldots & \frac{x_{n-1}}{x_{2}} \\
\vdots & \vdots & \vdots & \ddots & \vdots \\
\frac{1}{x_{n-1}} & \frac{x_{1}}{x_{n-1}} & \frac{x_{2}}{x_{n-1}} & \ldots & 1
\end{array}\right) \in \mathcal{P C} \mathcal{M}_{n}
$$

As it is apparent from matrix $\mathbf{A}_{\delta}$, such a simple perturbed PCM is constructed from a consistent PCM by entering a perturbation factor $\delta$ at its entry $a_{12}$, while its reciprocal entry $a_{21}$ is multiplied by $\frac{1}{\delta}$. We remark, that any location of the perturbed pair of elements in the PCM does not involve the loss of generality.

PCMs that can be made consistent by the modification of one/two/three entries have been analyzed by Bozóki, Fülöp and Poesz [4]. Out of 20 PCMs of size $4 \times 4$ originated from real decision problems, $6 \mathrm{PCMs}$ were simple perturbed. See [4, Table 1] for more findings. The more general idea of comparing two PCMs that differ from each other in a single entry (and its reciprocal) has been applied by Cook and Kress [8, Axiom 2] and also by Brunelli and Fedrizzi [5, Axiom 4].

Let $\lambda_{\delta}$ denote the Perron-eigenvalue of $\mathbf{A}_{\delta}$. It follows from (1) that if $\delta \neq 1$, then $\lambda_{\delta}>n$. Formally $\lambda_{1}=n$ holds, but we shall not consider $\mathbf{A}_{1}$ a simple perturbed PCM, as it is consistent.

Farkas [11] shows, that $\lambda_{\delta}$ can be obtained from the following equation:

$$
\lambda_{\delta}^{3}-n \lambda_{\delta}^{2}-(n-2)\left(\delta+\frac{1}{\delta}-2\right)=0 .
$$

One can write an explicit formula for $\lambda_{\delta}$ :

$$
\lambda_{\delta}=\frac{1}{6} \sqrt[3]{\frac{B+12 \sqrt{3 C}}{\delta}}+\frac{2}{3} \sqrt[3]{\frac{\delta}{B+12 \sqrt{3 C}}}+\frac{1}{3} n
$$

where

$$
\begin{aligned}
& B=8 n^{3} \delta+108 n \delta^{2}-216 n \delta+108 n-216 \delta^{2}+432 \delta-216 \\
& C=4 n^{4} \delta^{3}-8 n^{4} \delta^{2}+4 n^{4} \delta-8 n^{3} \delta^{3}+16 n^{3} \delta^{2}-8 n^{3} \delta+27 n^{2} \delta^{4}+162 n^{2} \delta^{2}
\end{aligned}
$$




$$
\begin{aligned}
& -108 n^{2} \delta^{3}-108 n^{2} \delta+27 n^{2}-108 n \delta^{4}+432 n \delta^{3}-648 n \delta^{2}+432 n \delta-108 n \\
& +108 \delta^{4}-432 \delta^{3}+648 \delta^{2}-432 \delta+108
\end{aligned}
$$

even if the main results of the paper can be proved without the expanded formula above. In the remainder of the paper $\lambda$ denotes $\lambda_{\delta}=\lambda_{\max }\left(\mathbf{A}_{\delta}\right)$.

Farkas, Rózsa and Stubnya [10] developed a general method to write the explicit form of the principal right eigenvector, when the perturbed elements are in the same row/column of the PCM. According to Farkas [11, Formula 26], the principal right eigenvector of the simple perturbed $\mathrm{PCM} \mathbf{A}_{\delta}$ can be written as

$$
\mathbf{w}^{E M}=\left(\begin{array}{c}
w_{1}^{E M} \\
w_{2}^{E M} \\
\vdots \\
w_{i}^{E M} \\
\vdots
\end{array}\right)=\left(\begin{array}{c}
\lambda-1+\delta \\
\frac{1}{x_{1}}\left(\lambda-1+\frac{1}{\delta}\right) \\
\vdots \\
\frac{1}{x_{i-1}} \lambda \frac{\lambda-2}{n-2} \\
\vdots
\end{array}\right), \quad i=3,4, \ldots, n
$$

The principal right eigenvector of $\mathrm{PCM} \mathbf{A}_{\delta}$ can be written in an alternative way (Farkas [11, Formula 24]):

$$
\mathbf{w}^{E M}=\left(\begin{array}{c}
w_{1}^{E M} \\
w_{2}^{E M} \\
\vdots \\
w_{i}^{E M} \\
\vdots
\end{array}\right)=c\left(\begin{array}{c}
\lambda(\lambda-n+1) \\
\frac{1}{x_{1}}\left[\lambda-\left(1-\frac{1}{\delta}\right)(\lambda-n+2)\right] \\
\vdots \\
\frac{1}{x_{i-1}}\left(\lambda-1+\frac{1}{\delta}\right) \\
\vdots
\end{array}\right), \quad i=3,4, \ldots, n
$$

where scalar $c$ can be expressed as $\frac{\lambda-1+\delta}{\lambda(\lambda-n+1)}$. Both formulas (5) -(6) shall be applied, depending on our purpose.

Remark 1. Every PCM of size $3 \times 3$ is either consistent or simple perturbed.

\section{Efficiency of the principal right eigenvector of a simple perturbed pairwise comparison matrix}

Note that the approximation of the entries of the bottom-right $(n-2) \times(n-2)$ submatrix of $\mathbf{A}_{\delta}$ is perfect, i.e., $a_{i j}=\frac{w_{i}^{E M}}{w_{j}^{E M}}(i, j=3,4, \ldots, n)$. It remains to check the approximations of the elements in the first and second rows and columns. 
Lemma 1. If $\delta>1$, then $\frac{w_{1}^{E M}}{w_{2}^{E M}}<a_{12}$. If $\delta<1$, then $\frac{w_{1}^{E M}}{w_{2}^{E M}}>a_{12}$.

Proof. Let $\delta>1$. From (5), the approximation of $a_{12}=\delta x_{1}$ is

$$
\frac{w_{1}^{E M}}{w_{2}^{E M}}=\frac{\lambda-1+\delta}{\frac{1}{x_{1}}\left(\lambda-1+\frac{1}{\delta}\right)}=x_{1} \frac{\lambda-1+\delta}{\lambda-1+\frac{1}{\delta}} .
$$

We shall prove that $\frac{\lambda-1+\delta}{\lambda-1+\frac{1}{\delta}}<\delta$, that is, $\lambda-1+\delta<\delta \lambda-\delta+1 \Leftrightarrow 2(\delta-1)<$ $\lambda(\delta-1) \Leftrightarrow 2<\lambda$. The last inequality holds, because $\lambda>n \geq 3$. The case $\delta<1$ is analogous.

Lemma 2. Let $j>2$. If $\delta>1$, then $\frac{w_{1}^{E M}}{w_{j}^{E M}}>a_{1 j}$. If $\delta<1$, then $\frac{w_{1}^{E M}}{w_{j}^{E M}}<a_{1 j}$.

Proof. Let $\delta>1$. From (6), the approximation of $a_{1 j}=x_{j-1}(j=3,4, \ldots, n)$ is

$$
\frac{w_{1}^{E M}}{w_{j}^{E M}}=\frac{\lambda(\lambda-n+1)}{\frac{1}{x_{j-1}}\left(\lambda-1+\frac{1}{\delta}\right)}=x_{j-1} \frac{\lambda(\lambda-n+1)}{\lambda-1+\frac{1}{\delta}} .
$$

The proposition is equivalent to $\frac{\lambda(\lambda-n+1)}{\lambda-1+\frac{1}{\delta}}>1 \Leftrightarrow \lambda(\lambda-n+1)>\lambda-1+\frac{1}{\delta} \Leftrightarrow$ $\lambda^{2}-\lambda n+\lambda>\lambda-1+\frac{1}{\delta} \Leftrightarrow\left(\lambda^{2}-\lambda n\right)+\left(1-\frac{1}{\delta}\right)>0$. The first expression is positive because $\lambda>n$, and the second one is positive because $\delta>1$.

Now let $\delta<1$. From (5) , the approximation of $a_{1 j}=x_{j-1}(j=3,4, \ldots, n)$ is

$$
\frac{w_{1}^{E M}}{w_{j}^{E M}}=\frac{\lambda-1+\delta}{\frac{1}{x_{j-1}} \lambda \frac{\lambda-2}{n-2}}=x_{j-1} \frac{(n-2)(\lambda-1+\delta)}{\lambda(\lambda-2)},
$$

the objective is to prove $(n-2)(\lambda-1+\delta)<\lambda(\lambda-2) \Leftrightarrow \lambda(n-\lambda)+(\delta-1)(n-$ $2)<0$. The first product is negative, because $\lambda>n$, the second product is also negative, because $\delta<1$.

Lemma 3. Let $j>2$. If $\delta>1$, then $\frac{w_{2}^{E M}}{w_{j}^{E M}}<a_{2 j}$. If $\delta<1$, then $\frac{w_{2}^{E M}}{w_{j}^{E M}}>a_{2 j}$.

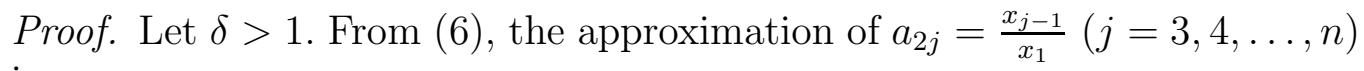
is

$$
\frac{w_{2}^{E M}}{w_{j}^{E M}}=\frac{\frac{1}{x_{1}}\left[\lambda-\left(1-\frac{1}{\delta}\right)(\lambda-n+2)\right]}{\frac{1}{x_{j-1}}\left(\lambda-1+\frac{1}{\delta}\right)}=\frac{x_{j-1}}{x_{1}} \frac{\lambda-\left(1-\frac{1}{\delta}\right)(\lambda-n+2)}{\lambda-1+\frac{1}{\delta}} .
$$

Thus, the proposition becomes equivalent to $\frac{\lambda-\left(1-\frac{1}{\delta}\right)(\lambda-n+2)}{\lambda-\left(1-\frac{1}{\delta}\right)} \frac{x_{j-1}}{x_{1}}<\frac{x_{j-1}}{x_{1}} \Leftrightarrow$ $\lambda-\left(1-\frac{1}{\delta}\right)(\lambda-n+2)<\lambda-\left(1-\frac{1}{\delta}\right) \Leftrightarrow-(\lambda-n+2)<-1 \Leftrightarrow \lambda-n>-1$, that holds, because $\lambda>n$. The proof of case $\delta<1$ is analogous to the reciprocals of the above described assertions. 
The next theorem states the main result of our paper:

Theorem 2. The principal right eigenvector of a simple perturbed pairwise comparison matrix is efficient.

\section{First proof:}

Suppose that $\delta>1$ and apply Lemmas 1, 2 and 3. Suppose there exists a weight vector $\mathbf{w}^{\prime}=\left(w_{1}^{\prime}, w_{2}^{\prime}, w_{3}^{E M}, w_{4}^{E M}, \ldots, w_{n}^{E M}\right)^{\top}$ that approximates matrix $\mathbf{A}_{\delta}$ at least as well as $\mathbf{w}^{E M}$ does, and strictly better than $\mathbf{w}^{E M}$ in one position. As is readily seen from Lemma 2, $w_{1}^{\prime} \leq w_{1}^{E M}$. Similarly, from Lemma 3, $w_{2}^{\prime} \geq w_{2}^{E M}$. At least one of these inequalities must be strict, otherwise $\mathbf{w}^{\prime}=\mathbf{w}^{E M}$. They, together with Lemma 1, imply that

$$
\frac{w_{1}^{\prime}}{w_{2}^{\prime}}<\frac{w_{1}^{E M}}{w_{2}^{E M}}<a_{12},
$$

therefore $\mathbf{w}^{\prime}$ provides a strictly worse approximation for $a_{12}$ than $\mathbf{w}^{E M}$ does, which contradicts the initial supposition. The case $\delta<1$ is analogous.

The First proof is simple and requires no prior knowledge in multiobjective optimization problems. Theorem 3.1. is explicitly based on matrix theory that provides a proof for the efficiency of the principal right eigenvector of PCMs with the specific structure of matrix $\mathbf{A}_{\delta}$. Additionally, by depicting the digraph representation of the studied problem one can easily visualize and check for such PCMs whether or not these solutions are, in fact, efficient.

\section{Second proof:}

Let us apply Definition 2 in order to draw the digraph associated to matrix $\mathbf{A}_{\delta}$ and its principal right eigenvector $\mathbf{w}^{E M}$. Namely, an arc goes from node $i$ to node $j$ if and only if $\frac{w_{i}^{E M}}{w_{j}^{E M}} \geq a_{i j}$. Let $\delta>1$. Lemma 1 implies that $(2 \rightarrow 1) \in \vec{E}$ and $(1 \rightarrow 2) \notin \vec{E}$. Lemma 2 implies that $(1 \rightarrow j) \in \vec{E}$ and $(j \rightarrow 1) \notin \vec{E}$ for all $j=3,4, \ldots, n$. Lemma 3 implies that $(2 \rightarrow j) \notin \vec{E}$ and $(j \rightarrow 2) \in \vec{E}$ for all $j=3,4, \ldots, n$. For $i, j=3,4, \ldots, n, i \neq j, \quad a_{i j}=\frac{w_{i}^{E M}}{w_{j}^{E M}}$ implies that $(i \rightarrow j) \in \vec{E}$ and $(j \rightarrow i) \in \vec{E}$. The digraph is drawn in Figure 2 . 


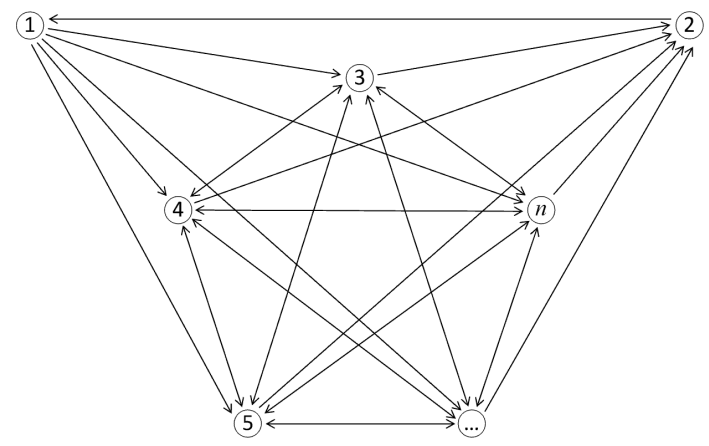

Figure 2. The strongly connected digraph of a simple perturbed pairwise comparison matrix $(\delta>1)$

The digraph is strongly connected, Theorem 1 can readily be used to show that the eigenvector $\mathbf{w}^{E M}$ is efficient. The case $\delta<1$ is similar: the corresponding digraph is the same as that of displayed in Figure 2 except that nodes 1 and 2 would be interchanged.

Our experiments have shown that the characterization of efficiency (Theorem 1) with the use of directed graphs (according to Definition 2) is, indeed, very robust and this approach seems to be applicable in more complicated cases, too.

Corollary 1. The principal right eigenvector of a $3 \times 3$ PCM is efficient.

Proof. The claim follows from Remark 1 and Theorem 2, It is worth noting that efficiency follows also from the equivalence of the eigenvector method and the row geometric mean, also known as the optimal solution to the logarithmic least squares problem [9, Section 3.2]. Blanquero, Carrizosa and Conde [2, Corollary 7] proved that the weight vector calculated by the row geometric mean is efficient.

\section{A numerical example}

Example 2. Let us choose $n=4, x_{1}=2, x_{2}=4, x_{3}=8, \delta=1.5$ in formula (4):

$$
\mathbf{A}_{1.5}=\left(\begin{array}{cccc}
1 & 3 & 4 & 8 \\
1 / 3 & 1 & 2 & 4 \\
1 / 4 & 1 / 2 & 1 & 2 \\
1 / 8 & 1 / 4 & 1 / 2 & 1
\end{array}\right)
$$


Matrix $\mathbf{A}_{1.5}$ is a simple perturbed PCM. Its principal right eigenvector $\mathbf{w}^{E M}$ and the consistent approximation of $\mathbf{A}_{1.5}$, generated by $\mathbf{w}^{E M}$, are displayed, truncated at 8 and 4 correct digits, respectively:

$\mathbf{w}^{E M}=\left(\begin{array}{c}0.57313428 \\ 0.23374121 \\ 0.12874966 \\ 0.06437483\end{array}\right), \quad\left[\frac{w_{i}^{E M}}{w_{j}^{E M}}\right]=\left(\begin{array}{cccc}1 & 2.4520 & 4.4515 & 8.9030 \\ 0.4078 & 1 & 1.8154 & 3.6309 \\ 0.2246 & 0.5508 & 1 & 2 \\ 0.1123 & 0.2754 & 1 / 2 & 1\end{array}\right)$.

One can verify that entry 3 in position (1,2) of $\mathbf{A}_{1.5}$ is underestimated by $\frac{w_{1}^{E M}}{w_{2}^{E M}}=2.4520$, in accordance with Lemma (11) and represented by the arc from node 2 to node 1 in Figure 2. Lemmas (2)-(3) can also be checked. Finally, entry 2 in position $(3,4)$ of $\mathbf{A}_{1.5}$ is estimated perfectly by $\frac{w_{3}^{E M}}{w_{4}^{E M}}=2$, represented by a bi-directed edge between nodes 3 and 4. The directed graph in Figure 2 is strongly connected, which ensures that the principal right eigenvector is efficient.

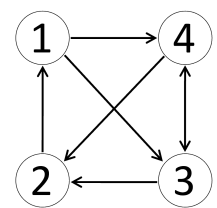

Figure 2. The principal right eigenvector in Example 5.1 is efficient, because its associated digraph is strongly connected

\section{Conclusions and open questions}

We have made a small step in the presumably long way of finding a necessary and sufficient condition of that the principal right eigenvector be efficient. It has been shown in the paper that if the PCM is simple perturbed, that is, it can be made consistent by a modification of an element and its reciprocal, then the principal right eigenvector is efficient. The explicit formulas of the principal right eigenvector of a simple perturbed PCM enabled us to provide a solid proof. We hope to return to a similar special case, when the PCM can be made consistent by a modification of two elements and their reciprocals. However, in the general case, explicit formulas do not exist, or, even if they exist, they may be hopelessly complicated.

Although we would not overrate the practical significance of simple perturbed PCMs, they do occur in real decision problems [4, Table 1]. On the 
other hand, they might help understanding the phenomenon of (in)efficiency, which is enigmatic at the moment. The question of the possible relations between the efficiency of the principal right eigenvector and the level of inconsistency is also to be investigated.

The authors believe that efficiency is a reasonable and desirable property, independently of the background of the analyst. Being an economist, engineer, decision theorist, preference modeler or mathematician, who faces an estimation problem, inefficient solutions are hard to argue for. The comparative studies of weighting methods, such as [1, 6, 9, 13], should be extended by adding efficiency to the list of axioms/criteria.

\section{Acknowledgments}

The authors would like to thank the three anonymous reviewers for their valuable and constructive recommendations. The authors are grateful to József Temesi (Department of Operations Research and Actuarial Sciences, Corvinus University of Budapest) for raising the idea of the efficiency analysis of simple perturbed pairwise comparison matrices. Comments of János Fülöp (Institute for Computer Science and Control, Hungarian Academy of Sciences (MTA SZTAKI) and Óbuda University, Budapest) and Örs Rebák (Corvinus University of Budapest) are highly appreciated. Research was supported in part by OTKA grant K 111797.

This paper is dedicated to the second author's grandmother, who celebrated her 100th birthday on September 15, 2015.

\section{References}

[1] G Bajwa, Eng Ung Choo, and William C Wedley. Effectiveness analysis of deriving priority vectors from reciprocal pairwise comparison matrices. Asia-Pacific Journal of Operational Research, 25(3):279-299, 2008.

[2] R. Blanquero, E. Carrizosa, and E. Conde. Inferring efficient weights from pairwise comparison matrices. Mathematical Methods of Operations Research, 64(2):271-284, 2006.

[3] S. Bozóki. Inefficient weights from pairwise comparison matrices with arbitrarily small inconsistency. Optimization, 63(12):1893-1901, 2014. 
[4] S. Bozóki, J. Fülöp, and A. Poesz. On pairwise comparison matrices that can be made consistent by the modification of a few elements. Central European Journal of Operations Research, 19(2):157-175, 2011.

[5] M. Brunelli and M. Fedrizzi. Axiomatic properties of inconsistency indices for pairwise comparisons. Journal of the Operational Research Society, 66:1-15, 2014.

[6] E.U. Choo and W.C. Wedley. A common framework for deriving preference values from pairwise comparison matrices. Computers $\&$ Operations Research, 31(6):893-908, 2004.

[7] Eduardo Conde and María de la Paz Rivera Pérez. A linear optimization problem to derive relative weights using an interval judgement matrix. European Journal of Operational Research, 201(2):537-544, 2010.

[8] W.D. Cook and M. Kress. Deriving weights from pairwise comparison ratio matrices: An axiomatic approach. European Journal of Operational Research, 37(3):355-362, 1988.

[9] Theo K. Dijkstra. On the extraction of weights from pairwise comparison matrices. Central European Journal of Operations Research, 21(1):103123, 2013.

[10] A. Farkas, P. Rózsa, and E. Stubnya. Transitive matrices and their applications. Linear Algebra and its Applications, 302-303:423-433, 1999.

[11] András Farkas. The analysis of the principal eigenvector of pairwise comparison matrices. Acta Polytechnica Hungarica, 4(2):99-115, 2007.

[12] Michele Fedrizzi. Obtaining non-dominated weights from preference relations through norm-induced distances. XXXVII Meeting of the Italian Association for Mathematics Applied to Economic and Social Sciences (AMASES), September 5-7, 2013, Stresa, Italy.

[13] B. Golany and M. Kress. A multicriteria evaluation of methods for obtaining weights from ratio-scale matrices. European Journal of Operational Research, 69(2):210-220, 1993.

[14] Thomas L. Saaty. A scaling method for priorities in hierarchical structures. Journal of Mathematical Psychology, 15(3):234-281, 1977.

[15] M. Zeleny. Multiple criteria decision making. McGraw-Hill, 1982. 